\title{
Predictive Factors for Recurrence of External Rectal Prolapse after Laparoscopic Ventral Rectopexy
}

\author{
Akira Tsunoda $^{1)}$, Tomoko Takahashi ${ }^{1)}$, Satoshi Matsuda ${ }^{2)}$ and Hiroshi Kusanagi ${ }^{1)}$ \\ 1) Department of Gastroenterological Surgery, Kameda Medical Center, Kamogawa, Japan \\ 2) Department of Pediatric Surgery, Kameda Medical Center, Kamogawa, Japan
}

\begin{abstract}
Objectives: This study aimed to evaluate factors that contribute to the recurrence of external rectal prolapse (ERP) following laparoscopic ventral rectopexy (LVR).

Methods: All patients who underwent LVR using synthetic meshes between 2011 and 2018 were prospectively included. A standard questionnaire including the Fecal Incontinence Severity Index (FISI) and Constipation Scoring System (CSS) was administered preoperatively and postoperatively. Defecography was performed 6 months postoperatively. Univariate and backward stepwise multivariate Cox analysis was performed to determine the prognostic factors of recurrence.

Results: In total, 132 patients with a median follow-up of 46 months were included. The overall recurrence rate was $6.8 \%(\mathrm{n}=9)$, as confirmed by defecography at 6 months in six of the patients. None of the patients developed mesh erosion. FISI and CSS scores were significantly reduced at 3 months and remained significantly reduced for 3 years. Multivariate analyses revealed that the predictors of recurrence included male sex (hazards ratio, 11.3; 95\% confidence interval, 3.0-43.0) and age $>80$ years (hazards ratio, 10.7; 95\% confidence interval, 1.3-86.3). Eight patients with recurrence underwent surgery via Delorme's procedure $(n=7)$ and posterior rectopexy $(n=1)$. Two patients with new-onset rectoanal intussusception and one with uncorrected sigmoidocoele underwent repeat LVR.

Conclusions: LVR is effective in treating ERP with low morbidity and low recurrence. Male patients and patients older than 80 years are at increased risk of recurrence. Hence, the LVR technique should be modified or coupled with other perineal procedures when treating ERP, especially in male patients.
\end{abstract}

\section{Keywords}

laparoscopic ventral rectopexy, external rectal prolapse, recurrence, risk factor

J Anus Rectum Colon 2021; 5(4): 376-385

\section{Introduction}

Laparoscopic ventral rectopexy (LVR) is increasingly recognized as a treatment of external rectal prolapse (ERP). LVR is associated with low morbidity and it not only controls the prolapse but also improves associated bowel symptoms, including fecal incontinence (FI) and obstructed defecation (OD)[1-4]. The improvement of quality of life (QOL) postoperatively has also been demonstrated previously[5,6].

The recurrence rate of ERP following LVR is generally low. A recent systematic review reported a prolapse recurrence of $0 \%-17 \%$ [7]. The cause of recurrence is multifactorial, and both technical failure and patient factors may contribute to recurrence. Inadequate anterior dissection and inadequate fixation of the mesh to the sacral promontory have been reported as relevant technical factors[8]. Fu et al.[9] re- 


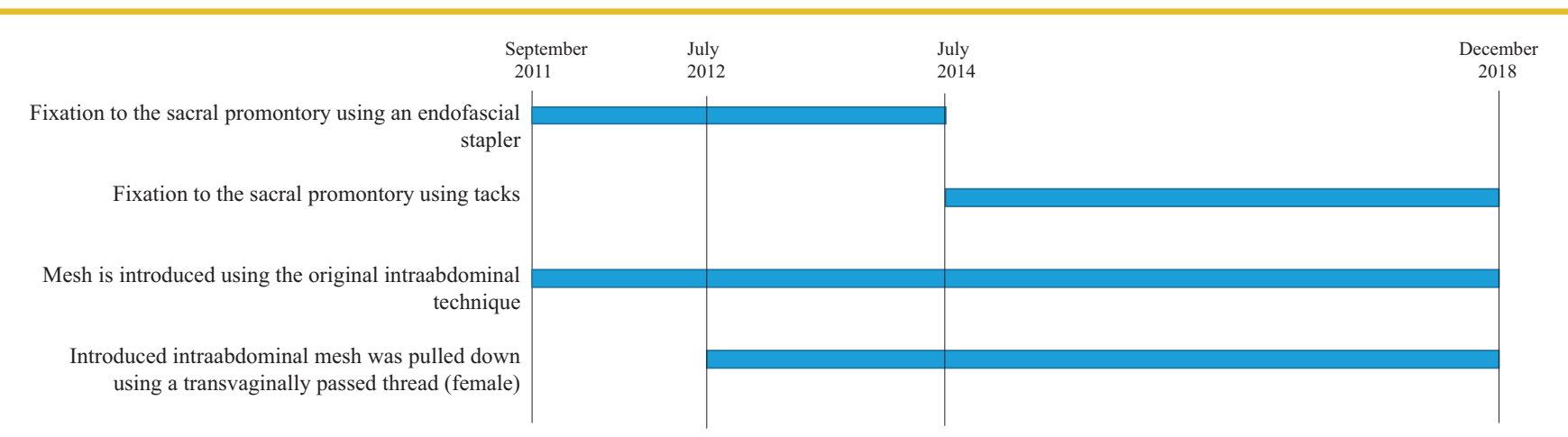

Figure 1. Technical chart illustrating the timeline of modifications to laparoscopic ventral rectopexy technique.

ported that recurrence was noted in 16/113 (14.2\%) female patients with ERP; additionally, using multivariate analysis they found that the use of a synthetic mesh for LVR was associated with higher recurrence. Meanwhile, the recurrence rate following LVR in male patients has been reported in a couple of studies with conflicting results $(0 \%$ vs. $17 \%)[10,11]$. Accordingly, the difference in the recurrence rates between the sexes and the effects of the sex on recurrence following LVR remains unclear. This study aimed to explore the factors contributing to the recurrence of ERP following LVR in both sexes using Cox regression analysis.

\section{Methods}

All consecutive patients who underwent LVR for ERP between September 2011 and December 2018 were prospectively enrolled. The present study group included the first 58 patients from our previous study[4].

The diagnosis of ERP was made clinically and, where this was not possible, based on evacuation proctography. Even if this was possible, proctography was performed in patients unless otherwise having severe symptoms or frailty, to assess the associated anatomical abnormalities.

During the same period, 16 patients underwent Delorme's procedure under spinal anesthesia because of major comorbid conditions that were contraindications for general anesthesia. All other patients with ERP were treated with LVR.

The patient characteristics recorded included the age, sex, body mass index (BMI), American Society of Anesthesiologists (ASA) classification, length of ERP, previous surgery for ERP or pelvic organ prolapse (POP), Fecal Incontinence Severity Index (FISI) score, and Constipation Scoring System (CSS) score. The surgical details included the method of sacral fixation, method of mesh introduction, operative time, blood loss, and postoperative complications. The length of postoperative stay was also recorded.

The ethical committee of Kameda Medical Center approved this study with a waiver of informed consent because of its retrospective nature (approval no. 20-108). Instead, information regarding the study protocol was made public, and patients were provided with the opportunity to withdraw consent. However, no patients or their relatives subsequently refused to participate in the study.

\section{Surgical technique}

All procedures were performed by or under the direct supervision of a single surgeon (A.T.) who is experienced in the operative techniques for LVR. The surgical procedure for LVR was performed as described by D'Hoore et al.[12].

Modifications to the technique were developed throughout the study period (Figure 1). This included the method of fixation of the polypropylene mesh to the sacral promontory, which initially included an endofascial stapler Endopath $^{\mathrm{TM}}$, EMS; Ethicon Endo-Surgery, Cincinnati, OH, USA), which was changed to titanium tacks (Autosuture Protack; Tyco Healthcare, Mansfield, MA, USA). Similarly, the method of introduction of the mesh was changed from the original intraabdominal technique[12] to our modified technique in female patients[13]. This technique includes passing a nylon thread with a straight needle by the perineal operator through the posterior wall of the vagina at the distal extent of dissection, which is caught in the abdominal cavity, extracted from the trocar, and fixed at the end of the mesh extracorporeally. The mesh is then introduced and pulled toward the pelvic floor using the nylon thread.

\section{Evacuation proctography}

A standardized proctography technique was used. To achieve this, the small bowel was opacified with a mixture containing $100 \mathrm{ml}$ of Barister ${ }^{\mathrm{TM}}$ (barium sulfate $100 \% \mathrm{w} / \mathrm{w}$; Fushimi Health Care Ltd., Japan) and $10 \mathrm{ml}$ of Urografin (60\% w/w; Bayer Pharmaceutical Ltd., Japan), which was ingested $2 \mathrm{~h}$ before the procedure. A synthetic stool containing barium sulfate, porridge oats and water (total of $150 \mathrm{ml}$ ) was inserted into the rectum using a 50-ml bladder syringe. Proctograms were evaluated according to the criteria described by Shorvon et al.[14]. Briefly, ERP was diagnosed when the full thickness of the rectum protruded the anal orifice. Rectoanal intussusception (RAI) was diagnosed when the apex of the rectal intussusception impinged on the inter- 
Table 1. Patient Demographics and Preoperative Characteristics.

\begin{tabular}{lc}
\hline Variable & \\
\hline Gender, $\mathrm{n}(\%)$ & $116(87.9)$ \\
$\quad$ Female & $16(12.1)$ \\
$\quad$ Male & $80.5(68.5-92.5)$ \\
Age, median (interquartile range), years & $66(50.0)$ \\
$\quad \leq 80$ years, $n(\%)$ & $66(50.0)$ \\
$>80$ years, $n(\%)$ & $21.8(17.2-26.4)$ \\
Body mass index, median (interquartile range) & \\
ASA physical status, $n(\%)$ & $10(7.6)$ \\
1 & $102(77.3)$ \\
2 & $20(15.2)$ \\
3 & $4.0(1.5-6.5)$ \\
Length of ERP, median (interquartile range), cm & $13(9.8)$ \\
Previous surgery for ERP, $n(\%)$ & $12 / 1$ \\
$\quad$ Perineal/abdominal & $10(7.6)$ \\
Previous surgery for POP, $n(\%)$ & $24(18.2)$ \\
Dementia/Schizophrenia, $n(\%)$ & $35(15-55)$ \\
FISI score, median (interquartile range) $(n=117)$ & $9(2-16)$ \\
CSS score, median (interquartile range) $(n=113)$ & \\
\hline
\end{tabular}

ERP, external rectal prolapse; FISI, Fecal Incontinence Severity Index; CSS, Constipation Scoring System

nal anal orifice or was intraanal based on images taken during maximal straining defecation. Rectorectal intussusception (RRI) was differentiated from RAI when the apex remained intrarectally and did not impinge on the internal anal orifice. Enterocoele or sigmoidocoele was diagnosed when the extension of the loop of the bowel was located between the vagina and rectum. Pelvic floor descent during defecation was estimated on the basis of the degree of the anorectal junction in relation to the inferior margin of the ischial tuberosity.

\section{Follow-up}

Patients were initially followed up at 3, 6, and 12 months and yearly thereafter. At each follow-up, FISI and CSS scores were calculated and a physical examination with a proctoscope was performed. An evacuation proctography was performed at 6 months. If a patient did not present for a follow-up, they were requested to report their FISI and CSS scores via phone or mail. Those who indicated that they had a feeling of prolapse were examined in the clinic. Recurrent ERP was diagnosed clinically and defined as a protrusion of the full-thickness of the rectum through the anal canal. Mucosal prolapse, in which there is a protrusion of only the rectal or anal mucosa, was not diagnosed as recurrent ERP. If this was not evident during the physical examination, proctography was repeated.

\section{Statistical analysis}

Quantitative data are presented as median with interquar- tile ranges unless otherwise specified. The analysis was performed using the Wilcoxon signed-rank test for paired data. Recurrence-free time was calculated as the time elapsed between the LVR surgery and clinical or radiological confirmation of anatomical recurrence. Recurrence-free survival was estimated using the Kaplan-Meier method, and survival curves were compared using a log-rank test. The Cox regression model was used to analyze the predictive factors for recurrence-free survival. Variables included in the analysis were age, sex, BMI, ASA physical status, previous surgery for ERP, length of ERP, sacral fixation, FISI score, and CSS score. The introduction of the mesh was excluded because the modified technique was adopted exclusively in female patients. The variables with moderate statistical associations $(P<0.2)$ with recurrence in univariate analysis were entered into backward stepwise multivariate analysis. The strength of these associations was quantified using the hazard ratio (HR) and 95\% confidence interval $(95 \% \mathrm{CI}) . P$ $<0.05$ was considered to indicate statistical significance. Statistical analysis was performed using SPSS v26 (IBM Corp., Armonk, NY, USA).

\section{Results}

Between September 2011 and December 2018, 132 patients underwent LVR. The overall median age was 81 years (69-93 years), and 116 (88\%) patients were women. The median duration of follow-up was 46.0 months (14.7-76.3 months). During the follow-up, $20(15.2 \%)$ patients died of causes unrelated to LVR at a median of 27 months (6-48 months) postoperatively without recurrent ERP. They died at the median age of 87 years (79-95 years). Table 1 summarizes the patient demographics and preoperative characteristics.

\section{Operative procedures and outcome}

The surgery was converted to open surgery in a single patient due to massive hemorrhage. There was no postoperative mortality. The median postoperative hospital stay was 1 day ( -1 to 3 days).

The overall postoperative complication rate was $3.8 \%$ (n $=5$ ). Two patients required surgery; one patient underwent laparoscopic adhesiolysis for small bowel obstruction during the primary surgery admission, whereas another patient was readmitted for port-site hernia repair. Additionally, 16 patients with POP underwent LVR and sacrocolpopexy (Table 2).

\section{Evacuation proctography}

Preoperative evaluation was performed in 80 patients. Findings other than ERP included enterocele in 34 women and sigmoidocoele in five women. Six months postoperatively, proctography was performed in 104 patients, includ- 
Table 2. Operative Procedures and Complications.

\begin{tabular}{lc}
\hline Variable & \\
\hline Sacral fixation method, $n(\%)$ & $46(34.8)$ \\
$\quad$ Endofascial stapler & $86(65.2)$ \\
Tacks & $16(12.1)$ \\
$\quad$ Colosuspension & \\
Introduction of mesh, $n(\%)$ & $36(27.3)$ \\
$\quad$ Intraabdominal alone & $96(72.7)$ \\
$\quad$ Modified technique (female patients)* & $182(103-261)$ \\
Operative time, median (interquartile range), min & $10(5-15)$ \\
Blood loss, median (interquartile range), ml & $1(-1$ to 3) \\
Postoperative hospital stay, median (interquartile range), days & $5(3.8)$ \\
Surgical complications, $n(\%)$ & 2 \\
$\quad$ Wound infection & 1 \\
$\quad$ Port site hernia & 1 \\
Small bowel obstruction & 1 \\
\hline Small bowel injury & \\
\hline
\end{tabular}

* Introduced intraabdominal mesh was pulled down using a trans-vaginally passed thread.

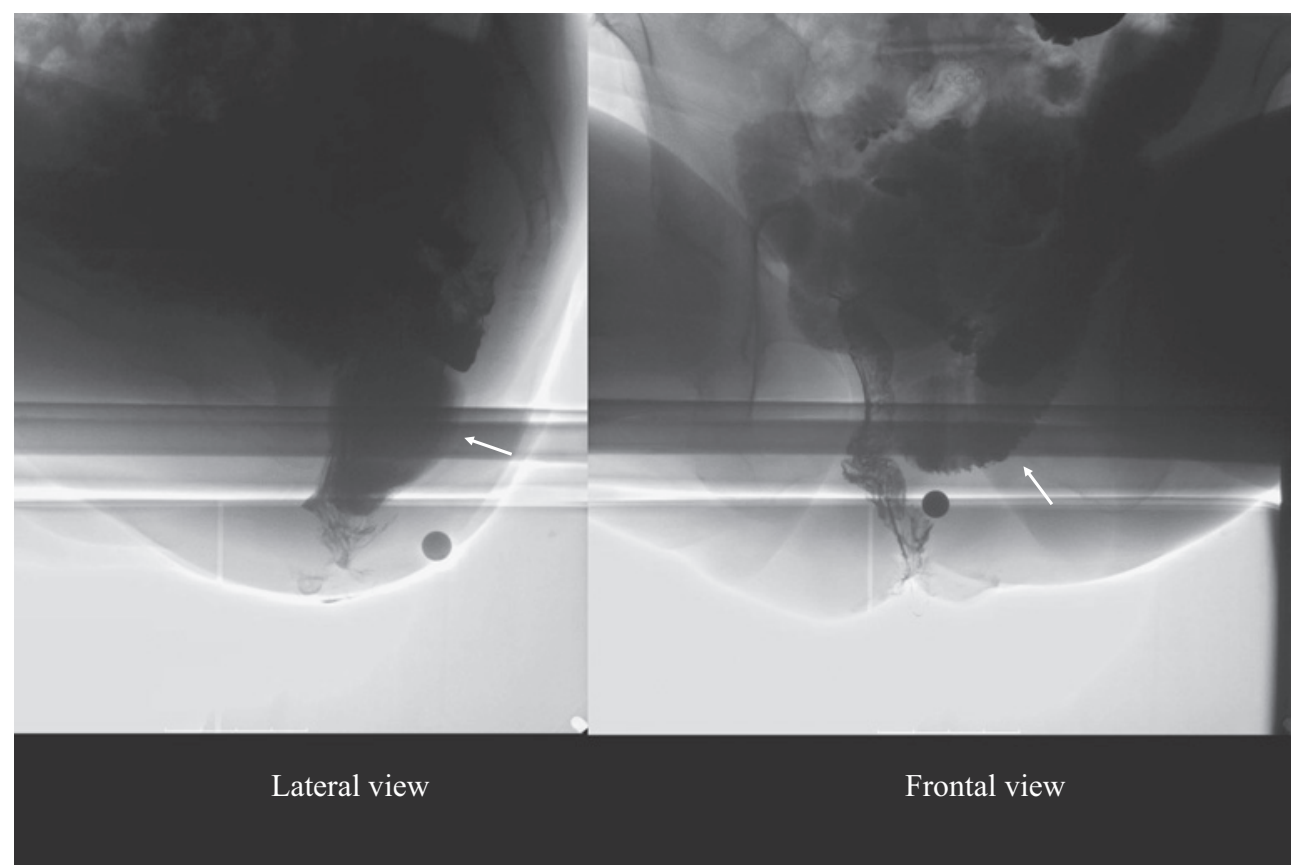

Figure 2. Postoperative proctography showing a so-called pararectal hernia (arrow).

ing 75 patients who were evaluated preoperatively. The findings revealed recurrent ERP in six patients. ERP was replaced by RAI in 22 patients and RRI in nine patients. Enterocoele disappeared in 34 patients but the site of herniation of the small bowel moved posteriorly to a point along the rectum in six patients (pararectal hernia) (Figure 2). Sigmoidocoele did not disappear in one of the five patients. The median pelvic floor descent was not significantly reduced (preoperatively, 22.7 vs. postoperatively, $18.9 \mathrm{~mm} ; P$ $=0.30)($ Table 3$)$.

\section{Clinical outcome}

The FISI score was assessed preoperatively in 117 patients after excluding 15 patients with senile dementia or schizophrenia. The median FISI score was significantly reduced at 3 months (preoperatively, 35 vs. postoperatively, $12 ; P<0.0001)$. This improvement was sustained for 3 years. CSS score was not assessed preoperatively in 19 patients including the aforementioned 15 patients with psychiatric disorders. The median CSS score was significantly reduced at 3 months (preoperatively, 9 vs. postoperatively, 4.5; 
Table 3. Evacuation Proctography Findings.

\begin{tabular}{|c|c|c|}
\hline & Preop $(n=80)$ & At 6 months $(n=104)$ \\
\hline External rectal prolapse & 80 & 6 \\
\hline Rectoanal intussusception & 0 & 22 \\
\hline Rectorectal intussusception & 0 & 9 \\
\hline Enterocele & 34 & 0 \\
\hline Sigmoidocele & 5 & 1 \\
\hline Pararectal hernia $^{\dagger}$ & 0 & 6 \\
\hline Pelvic floor descent ${ }^{\#}$, median (interquartile range), $\mathrm{mm}(n=75)$ & $22.7(8.0-37.4)$ & $18.9(1.2-36.6) *$ \\
\hline
\end{tabular}

$P<0.0001$ ), and the improvement was sustained for 3 years (Figure 3). The median age of 16 male patients was 74 years (42-106 years). Among the five male patients who were less than 60 years old, three had psychiatric disease. The remaining two patients (41 and 59 years old) reported that their postoperative sexual function was normal.

The overall cumulative recurrence rate of ERP following LVR was $6.8 \%(\mathrm{n}=9)$. The recurrence was found in five of $16(31.2 \%)$ male patients and four of $116(3.4 \%)$ female patients. Using Kaplan-Meier survival analysis, the overall 5year recurrence-free survival was $93.1 \%$ (Figure 4). All recurrences occurred within the first 12 months of surgery with a recurrence rate of $88.9 \%(n=8)$ and $11.1 \%(n=1)$ at 14 months.

\section{Reoperation}

Eight patients underwent surgery because of recurrent ERP; seven underwent Delorme's procedure and one underwent posterior mesh rectopexy. Additionally, two of the 22 patients with new-onset RAI who had persistent FI and one patient with uncorrected sigmoidocoele underwent laparoscopic surgery. Revisional surgery revealed that the mesh fixed using an endofascial stapler was detached from the sacrum; LVR was repeated using another mesh fixed using tacks at the sacral promontory in these cases. Six patients underwent transanal excision for mucosal prolapse. None of the patients had further mesh-related complications (Table 4).

\section{Risk factors for recurrence}

In the univariate analysis, the variables associated with recurrence were age, sex, and sacral fixation. Further backward stepwise multivariate Cox regression analysis revealed that age and sex were independent factors associated with a significantly increased risk of recurrence (male sex: HR = 11.3, 95\%CI, 3.0-43.0; age $>80$ years: $\mathrm{HR}=10.7,95 \% \mathrm{CI}$, 1.3-86.3) (Table 5). There was no significant difference in the recurrence rate between using an endofascial stapler and using tacks or between the methods of introduction of the mesh; the intraabdominal alone and the modified technique. The Kaplan-Meier curves comparing male patients with female patients and older patients with younger patients are presented in Figure 5, 6. At 5 years after the surgery, recurrence-free survival was $97.3 \%$ in female patients vs. $68.8 \%$ in male patients $(P<0.0001)$ and $98.5 \%$ in younger patients vs. $87.7 \%$ in older patients $(P=0.015)$.

\section{Discussion}

The recurrence of ERP following LVR is multifactorial, and the causes include both technical and clinical factors. Inadequate anterior rectal dissection and inadequate fixation of the mesh to the anterior rectal wall or sacral promontory and the type of mesh used are some of the reported technical factors $[8,15]$. Furthermore, sex, BMI, and previous history of prolapse repair[9,11] have been suggested as the clinical factors. Nonetheless, these studies did not determine the risk factors for recurrence using multivariate analysis except that by $\mathrm{Fu}$ et al.[9] who evaluated the factors contributing to recurrence in female patients using a Cox hazard regression model. Using the same model in the present cohort that included both sexes, we found that male patients and patients older than 80 years old are at increased risk of recurrence following LVR.

A couple of studies have provided conflicting results on the recurrence of ERP following LVR in male patients. Owais et al.[10] did not observe recurrence in 18 patients with ERP, whereas Rautio et al.[11] observed recurrence in nine $(17 \%)$ of 52 male patients. A systematic review reported that male patients may potentially have an effect on the recurrence of ERP[7]. The recurrence rate of ERP following LVR was estimated to be $6.9 \%$ at 5 years in this study, which is comparable with rates reported previously (0\%-17\%)[7]. However, male patients in this study had a high recurrence rate $(31 \%)$. LVR appears to be technically demanding in male patients with a narrow pelvis compared 

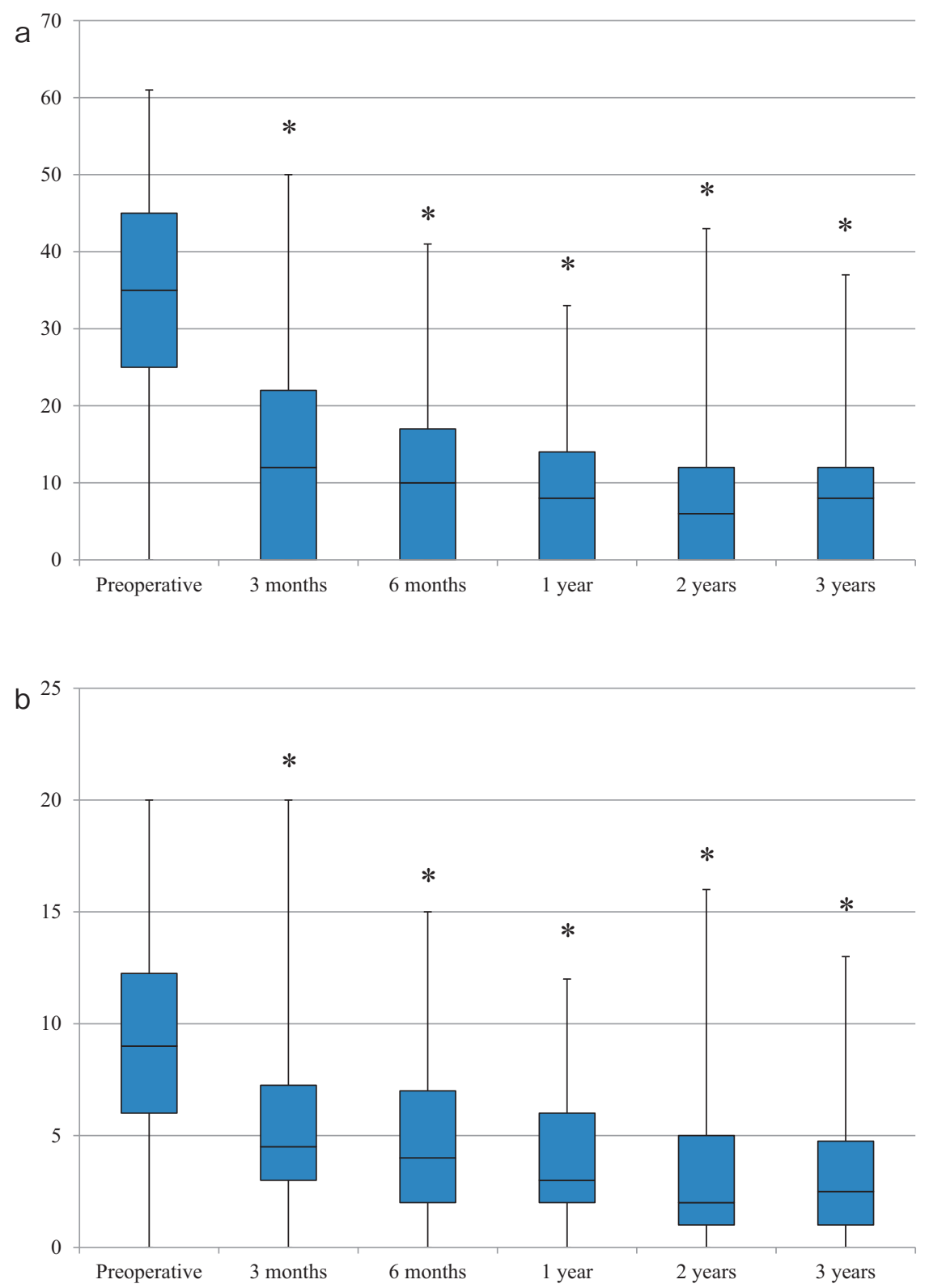

Figure 3.

a: Fecal Incontinence Severity Index score.

b: Constipation Scoring System score.

Boxes show median values with upper and lower quartiles. The vertical line extends from the minimum to the maximum values. $* P<0.0001$ versus preoperative.

with that in female patients, and complete dissection of the rectoprostatic septum down to the pelvic floor might be inadequate. Thus, the site of the fixed mesh in our male patients might not have been as distal as that in our female patients (a median distance of $2 \mathrm{~cm}$ from the ostium of the vagina). This can be a possible cause of recurrence in male patients. Another factor may be the detachment of staples from the promontory, possibly, because of stronger straining effort during defecation in men than that in women. However, we had insufficient evidence because mesh detachment was confirmed on a revisional survey in one out of five male patients with recurrence.

We found that the age of $>80$ years was independently associated with the risk of recurrence. A recent study also reported, using univariate analysis, that age of $>70$ years was a prognostic factor of recurrence[9]. This may be caused by a weaker pelvic floor and poor anal sphincter function in older persons. Previous studies have reported that aging is correlated not only with pelvic floor weakness[16] but also with lower anal sphincter function[17]. In fact, low anal resting and squeeze pressure in older patients with ERP has been reported[18]. Fu et al.[9] also found that prolonged pu- 
Recurrence-free (\%)

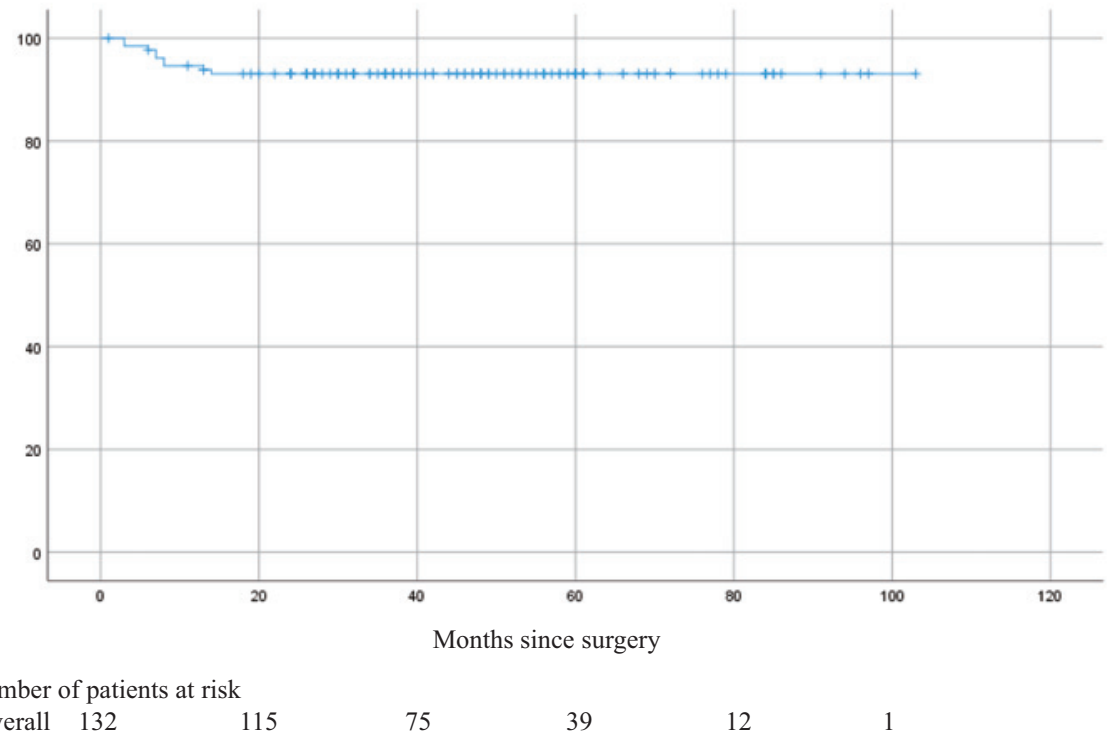

Figure 4. Kaplan-Meier analysis of recurrence-free survival following laparoscopic ventral rectopexy in patients with external rectal prolapse.

Table 4. Additional Procedures Performed for Recurrence after LVR or Persistent Symptoms.

\begin{tabular}{lc}
\hline & No. of patients $(n=17)$ \\
\hline Delorme's procedure & 7 \\
Redo LVR & 3 \\
Posterior mesh rectopexy & 1 \\
Transanal mucosectomy & 6 \\
\hline
\end{tabular}

dendal nerve terminal motor latency, which indicates denervation of the external anal sphincter, was predictive of recurrence.

Obesity is recognized as a risk factor for a higher recurrence rate following LVR because of a demanding pelvic dissection[19]. A recent review article demonstrated three studies that included patients with mean BMI $>25 \mathrm{~kg} / \mathrm{m}^{2}$ who had higher ERP recurrence rates than that in three other studies that included patients with lower BMI[7]. Additionally, another study reported that higher BMI (27.73 vs. $24.4 \mathrm{~kg} / \mathrm{m}^{2}$ ) led to worse outcomes following LVR in patients with OD[20]. Our patients, with a median BMI of 22 $\mathrm{kg} / \mathrm{m}^{2}$, were not obese, and we did not find high BMI to be a significant risk factor of recurrence.

Previous surgery for ERP was not correlated with the risk of recurrence in this study. Of 13 patients who underwent LVR for recurrent ERP after a failed procedure, full thickness recurrence occurred in a single male patient $(7.7 \%)$. Previous studies[2,9] also found that the efficacy of LVR for recurrent prolapse following a failed perineal or abdominal procedure is similar to that of primary LVR.
The type of mesh may have an influence on the risk of recurrence following LVR as well. Fu et al.[9] found that the use of synthetic meshes was correlated with a higher recurrence rate. By contrast, a review article found that 12 studies that used only synthetic meshes had a lower recurrence rate than that in three other studies that used either synthetic or biological meshes or only biological meshes[7]. In this study, we used a synthetic polypropylene mesh in all patients. Although our patients did not experience meshrelated vaginal or rectal erosion, a previous multiinstitutional study demonstrated that biological meshes may be associated with a lower incidence of mesh erosion compared with that in synthetic meshes[21].

The method of fixation of the mesh to the sacral promontory appears to be an important factor for recurrence. Twelve studies that used tacks only for fixation of the mesh to the promontory reported lower recurrence rates than those in three studies that used either tacks, screws, or sutures[7]. We did not find a significant difference in the recurrence rate between using an endofascial stapler and using tacks.

We had previously reported the functional and QOL assessments in 58 consecutive patients from this series[4]. The current study confirmed the improvements in FI and OD postoperatively, which is consistent with the results of previous studies[1,2,5].

Postoperative proctography showed that enterocele was eliminated in all affected patients, whereas a pararectal hernia developed in six patients. This may be because the left side of the deep pouch of Douglas was left intact, despite the rectovaginal septum being obliterated by the mesh up to the vaginal cervix, and the J-shaped incisional peritoneum 
Table 5. Risk Factors Affecting Recurrence.

\begin{tabular}{lcccc}
\hline & \multicolumn{2}{c}{ Univariate Cox regression } & \multicolumn{2}{c}{$\begin{array}{c}\text { Backward stepwise } \\
\text { multivariate Cox regression }\end{array}$} \\
\cline { 2 - 5 } & HR $(95 \%$ CI $)$ & $P$ & HR $(95 \%$ CI $)$ & $P$ \\
\hline Male & $10.17(2.73-37.90)$ & 0.001 & $11.32(2.98-43.01)$ & $<0.0001$ \\
Age $(>80$ years $)$ & $8.44(1.06-67.50)$ & 0.04 & $10.73(1.33-86.33)$ & 0.026 \\
Body mass index $(\geqq 22)$ & $2.16(0.54-8.65)$ & 0.28 & & \\
ASA Physical Status 3 & $0.79(0.10-6.34)$ & 0.83 & & \\
Previous surgery for ERP & $1.12(0.14-8.97)$ & 0.91 & & \\
Length of ERP $(>4$ cm) & $2.09(0.56-7.77)$ & 0.27 & & \\
Sacral fixation $($ tacks $)$ & $4.40(0.55-35.17)$ & 0.16 & $0.28(0.04-2.30)$ & \\
FISI score $(>34)(n=117)$ & $0.99(0.27-3.69)$ & 0.99 & & \\
CSS score $(\geqq 9)(n=113)$ & $0.96(0.27-3.56)$ & 0.95 & & \\
\hline
\end{tabular}

ERP, external rectal prolapse; FISI, Fecal Incontinence Severity Index; CSS, Constipation Scoring System

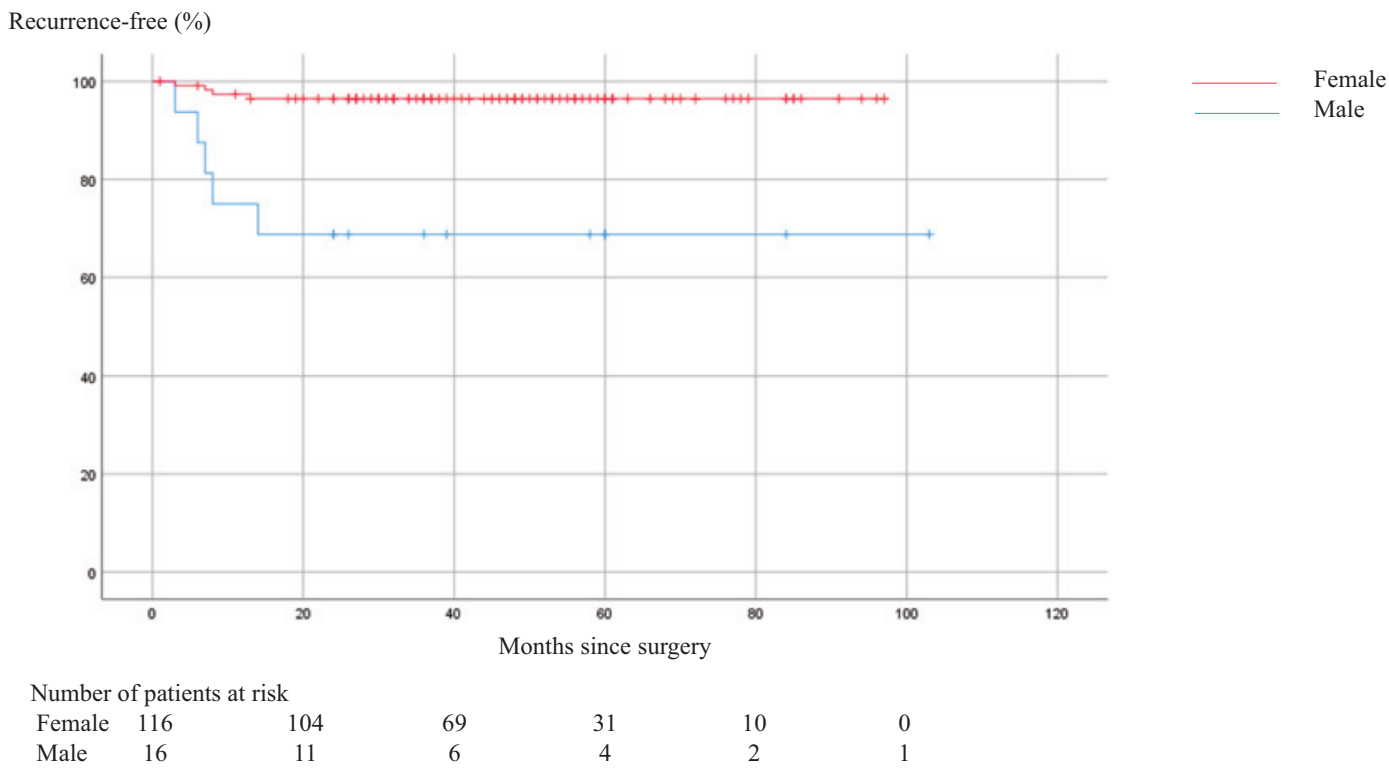

Figure 5. Kaplan-Meier analysis of recurrence-free survival following laparoscopic ventral rectopexy for external rectal prolapse in male and female patients.

was closed over the mesh.

In this study, 20 patients died of causes unrelated to LVR at the median age of 87 years (79-95 years) postoperatively. The influence of LVR in elderly patients with ERP on shortening their lives is unclear. Perineal procedures may not affect their lifetime. Further studies are required to determine whether a specific surgical procedure may have an effect on postoperative life in elderly patients.

The present study has certain limitations. First, there were differences in the follow-up between patients. Our patients were 10 years older than those in the previous studies[1-3,9,11], and $15 \%$ of our patients died of causes unrelated to LVR during the follow-up. Second, the method of introducing the mesh was not the same between the sexes; the intraabdominal mesh was pulled down using a transvagi- nally passed thread in most of the female patients. Third, some technique changes have been made during the study period. These limitations should be considered when interpreting the risk factors for recurrence following LVR.

To conclude, the male sex and age $>80$ years are independent factors for recurrence of ERP following LVR. Thus, the LVR technique should be modified or coupled with other perineal procedures when treating ERP, especially in male patients and older patients. Further studies are needed to validate the results of the present study.

\section{Acknowledgements}

The authors thank Yuko Tsunoda for her assistance with the statistical analysis. We would like to thank Editage (www.editage.jp) for the English editing. 
Recurrence-free (\%)
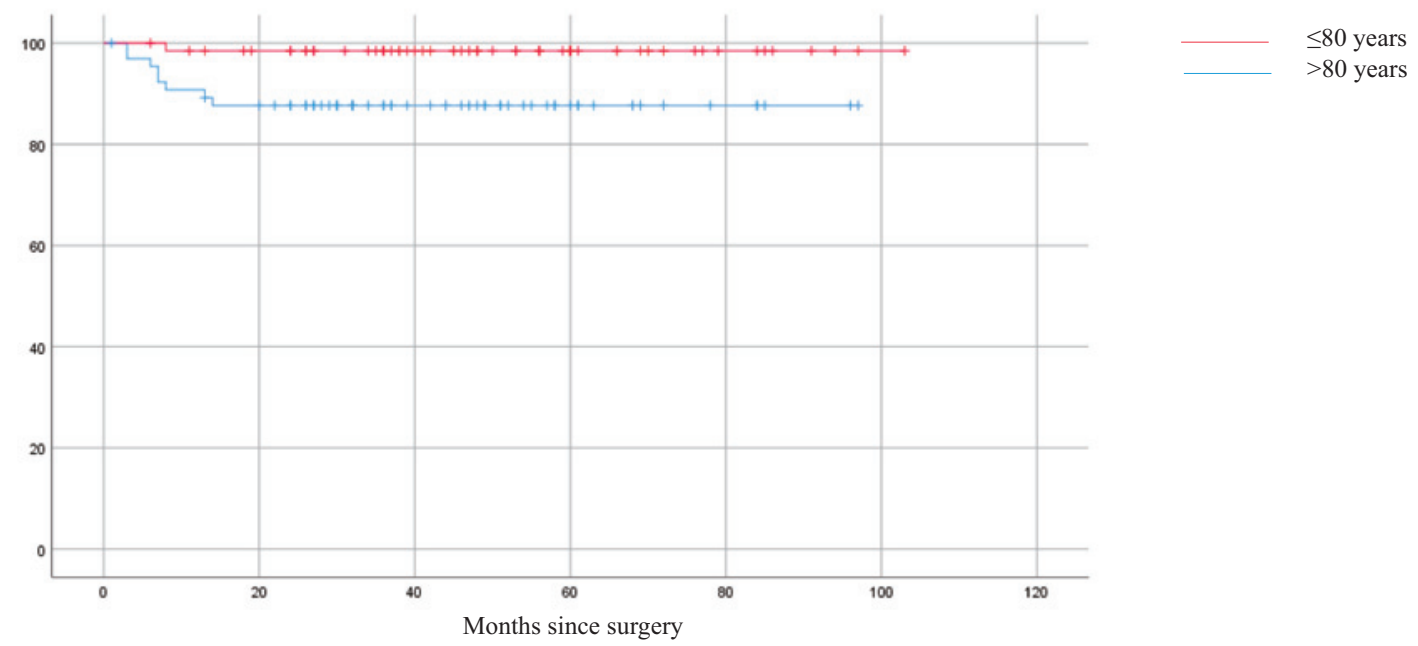

Number of patients at risk

$\begin{array}{lllllll}\leq 80 \text { years } & 66 & 60 & 42 & 23 & 7 & 1 \\ >80 \text { years } & 66 & 55 & 33 & 15 & 5 & 0\end{array}$

Figure 6. Kaplan-Meier analysis of recurrence-free survival following laparoscopic ventral rectopexy for external rectal prolapse in older ( $>80$ years) and younger $(\leq 80$ years $)$ patients.

\section{Conflicts of Interest}

There are no conflicts of interest.

\section{Author Contributions}

Akira Tsunoda: the design of the research, acquisition of data, analysis and interpretation of data, drafting of the article, and final approval of the version to be published.

Tomoko Takahashi: acquisition of data, analysis and interpretation of data, critical revision of the article for important intellectual content, and final approval of the version to be published.

Satoshi Matsuda: the design of the research, analysis and interpretation of data, critical revision of the article for important intellectual content, and final approval of the version to be published.

Hiroshi Kusanagi: the design of the research, analysis and interpretation of data, critical revision of the article for important intellectual content, and final approval of the version to be published.

Approval by Institutional Review Board (IRB)

Institutional review board: Ethical Committee of Kameda Medical Center

Review board approval number: 20-108

\section{References}

1. Consten EC, van Iersel JJ, Verheijen PM, et al. Long-term outcome after laparoscopic ventral mesh rectopexy: an observational study of 919 consecutive patients. Ann Surg. 2015 Nov; 262(5): 742-7.

2. Randall J, Smyth E, McCarthy K, et al. Outcome of laparoscopic ventral mesh rectopexy for external rectal prolapse. Colorectal Dis. 2014 Nov; 16(11): 914-9.

3. Faucheron JL, Voirin D, Riboud R, et al. Laparoscopic anterior rectopexy to the promontory for full-thickness rectal prolapse in 175 consecutive patients: short- and long-term follow-up. Dis Colon Rectum. 2012 Jun; 55(6): 660-5.

4. Tsunoda A, Takahashi $T$, Matsuda $S$, et al. Midterm functional outcome after laparoscopic ventral rectopexy for external rectal prolapse. Asian J Endosc Surg. 2020 Jan; 13(1): 25-32.

5. Maggiori L, Bretagnol F, Ferron M, et al. Laparoscopic ventral rectopexy: a prospective long-term evaluation of functional results and quality of life. Tech Coloproctol. 2013 Aug; 17(4): 431-6.

6. Tsunoda A, Takahashi T, Ohta T, et al. Quality of life after laparoscopic ventral rectopexy. Colorectal Dis. 2016 Aug; 18(8): O30110.

7. Emile SH, Elfeki H, Shalaby M, et al. Outcome of laparoscopic ventral mesh rectopexy for full-thickness external rectal prolapse: a systematic review, meta-analysis, and meta-regression analysis of the predictors for recurrence. Surg Endosc. 2019 Aug; 33(8): 2444-55.

8. Badrek-Al Amoudi AH, Greenslade GL, Dixon AR. How to deal with complications after laparoscopic ventral mesh rectopexy: lessons learnt from a tertiary referral centre. Colorectal Dis. 2013 Jun; 15(6): 707-12.

9. Fu CW, Stevenson AR. Risk factors for recurrence after laparoscopic ventral rectopexy. Dis Colon Rectum. 2017 Feb; 60(2): 178-86.

10. Owais AE, Sumrien H, Mabey K, et al. Laparoscopic ventral mesh rectopexy in male patients with internal or external rectal prolapse. Colorectal Dis. 2014 Dec; 16(12): 995-1000.

11. Rautio T, Mäkelä-Kaikkonen J, Vaarala M, et al. Laparoscopic ventral rectopexy in male patients with external rectal prolapse is associated with a high reoperation rate. Tech Coloproctol. 2016 Oct; 20(10): 715-20. 
12. D'Hoore A, Cadoni R, Penninckx F. Long-term outcome of laparoscopic ventral rectopexy for total rectal prolapse. Br J Surg. 2004 Nov; 91(11): 1500-5.

13. Tsunoda A, Takahashi $T$, Ohta $T$, et al. A novel technique of introducing the mesh at the distal dissection while performing laparoscopic ventral rectopexy. Colorectal Dis. 2016 Sep; 18(9): O334-6.

14. Shorvon PJ, McHugh S, Diamant NE, et al. Defecography in normal volunteers: results and implications. Gut. 1989 Dec; 30(12): 1737-49.

15. Mackenzie H, Dixon AR. Proficiency gain curve and predictors of outcome for laparoscopic ventral mesh rectopexy. Surgery. 2014 Jul; 156(1): 158-67.

16. Lewicky-Gaupp C, Brincat C, Yousuf A, et al. Fecal incontinence in older women: are levator ani defects a factor? Am J Obstet Gynecol. 2010 May; 202(5): 491.e1-6.

17. Pretlove SJ, Radley S, Toozs-Hobson PM, et al. Prevalence of anal incontinence according to age and gender: a systematic review and meta-regression analysis. Int Urogynecol J Pelvic Floor Dysfunct.
2006 Jun; 17(4): 407-17.

18. Tsunoda A, Yasuda N, Yokoyama N, et al. Delorme's procedure for rectal prolapse: clinical and physiological analysis. Dis Colon Rectum. 2003 Sep; 46(9): 1260-5.

19. Mercer-Jones MA, D'Hoore A, Dixon AR, et al. Consensus on ventral rectopexy: report of a panel of experts. Colorectal Dis. 2014 Feb; 16(2): 82-8.

20. Kremel D, Riss S, Müller C, et al. Adverse obstetric history is not a risk factor for poor outcome after ventral rectopexy for obstructive defaecation syndrome. Colorectal Dis. 2018 Dec; 20(12): 1125-31.

21. Evans C, Stevenson AR, Sileri P, et al. A multicenter collaboration to assess the safety of laparoscopic ventral rectopexy. Dis Colon Rectum. 2015 Aug; 58(8): 799-807.

Journal of the Anus, Rectum and Colon is an Open Access journal distributed under the Creative Commons Attribution-NonCommercial-NoDerivatives 4.0 International License. To view the details of this license, please visit (https://creativ ecommons.org/licenses/by-nc-nd/4.0/). 